\title{
BRI1 Signaling in the Root is Mediated through the SERK1 and SERK3 Co-receptors
}

\section{Anh Phu Nam Bui}

Faculty of Biotechnology, Ho Chi Minh City Open University, 35 Ho Hao Hon Street, Ho Chi Minh City, Vietnam

\begin{abstract}
Brassinosteroid (BR) is a class of polyhydroxysteroids plant hormones known to regulate shoot and root growth. Genetic and molecular analyses demonstrate that receptor kinase BRI1 protein acts as a perceiver for BR. One of the characteristics of bril mutant's phenotypes is the complete BR insensitivity in the root. Biochemical evidences of the BRI1 protein complex indicates that somatic embryogenesis receptor kinase 1 (SERK1) and SERK3 participate in the BR pathway in Arabidopsis root. While only serk3 mutants show partial reduction to BR sensitivity, serk1 presents a normal BR penetration phenotype compared to the wild type. Interestingly, the double mutant serk1serk3 displays more, but not full resistance to BR in root length assay. In this study, we aimed to enhance the BR insensitivity of the double mutant serk1serk3 by crossing serk1 mutant allele with a strong serk3 and bri1 mutant alleles. In our study, by generating serk1-3serk3-2 double mutants, a complete insensitivity to BR that phenocopied bri1-301 mutant was recorded. However, we were unable to increase BR resistance in the root of serk1-3serk3-2 double mutant by crossing with bril mutant allele in the triple mutant serk1-3serk3-2bril. As a result, all the BRI1 signaling in the root was mediated through the SERK1 and SERK3 co-receptors. Additionally, we established that based on conventional BR assays, the At1g27190 protein was also involved in BR signaling. Preliminary data indicated that the triple mutant serk1serk3-2At1g27190 showed a dwarfed phenotype. Whether or not this dwarfed phenotype is linked to BRI1

ARTICLE INFO

Article history:

Received: 19 May 2020

Accepted: 01 September 2020

Published: 27 November 2020

DOI: https://doi.org/10.47836/pjtas.43.4.13

E-mail address:

buiphunamanh@yahoo.com signaling impairment needs to be further investigated.

Keywords: At1g27190, brassinosteroid, brassinosteroid insensitive, somatic embryogenesis receptor kinase
\end{abstract}




\section{INTRODUCTION}

Brassinosteroids (BRs) are essential regulators of plant growth and development affecting a broad spectrum of processes at the molecular, cellular, and physiological levels (Fan et al., 2016). The importance of BR in plant development is illustrated by the dramatic phenotypes of bril (brassinosteroid insensitive) mutant plants failing to produce or perceive BR (Bücherl et al., 2013). These bri 1 mutants are characterized by short roots, dwarfed stature, male sterility, rounded leaves, and photo-morphogenetic defects (Kang et al., 2017; Sun et al., 2017). Major progresses in understanding the BR signaling cascade have been performed (Belkhadir \& Chory, 2006; Belkhadir \& Jaillais, 2015). However, considering the highly pleiotropic defects of the bril mutants and the ubiquitous expression of bril gene, it remains to be established how BR specificity can be achieved in this signaling system (Hutten et al., 2017).

Some models suggest the involvement of interacting partner proteins for BRI1. A gain-of-function screen identified a leucine rich repeat-receptor like kinase (LRRRLK) protein, somatic embryogenesis receptor-like kinase 3 (SERK3) (Brandt \& Hothorn, 2016; Santiago et al., 2013; Sun et al., 2013), which hetero-dimerizes with BRI1 (Russinova et al., 2004). Since the formation of the SERK3-BRI1 complex is BR-dependent (Kinoshita et al., 2005; Wang et al., 2005), SERK3 is suggested to play a key role in downstream signaling events in BR-dependent manner. Another line of biochemical evidences also indicates that SERK1 contributes to the BR pathway (Albrecht et al., 2012; Karlova et al., 2006; van Esse et al., 2012). SERK1 and SERK3 are parts of a large family of 13 SERK-like receptors with 5 closely related members (SERK1 to SERK5) (Liu et al., 2020). The mild phenotypes of the single mutants serk3 and serk1 indicate that other SERK members could participate in the BRs signaling as well (Zheng et al., 2018). These findings have shed lights that spatial and temporal expression of SERK-proteins might be responsible for the specificity of the BRs signaling (van Esse et al., 2016).

Due to the redundancy in the SERK protein family, it remains debatable whether all responses of the BRI1 signaling are mediated through SERKs receptors (van Esse et al., 2013). Using a set of double, triple and quadruple mutants within the subfamily SERK1 to SERK5, the results exhibit that only serkl enhances the BR insensitivity of serk 3 mutant roots. However, data also indicates that SERK1 and SERK3 partially account for the BR insensitivity root phenotype, i.e. the double serk1serk3 mutant does not show complete insensitivity as bril mutants do (Albrecht et al., 2008).

There have been several hypotheses to explain the various penetrations of BR sensitivity phenotype in the root. Firstly, serk 3 mutant allele used in the previous studies is not the strongest allele (Albrecht et al., 2008, 2012; Kemmerling et al., 2007). Secondly, there could be other co-receptors than the SERK are involved in BR signaling cascade. We identified in the SERK3 and SERK1 protein complex, another LRR- 
RLK, At1g27190 (unpublished data). Finally, an alternative BRI1 pathway that does not utilize of the SERK co-receptors is also proposed.

In our study, we established that the level of BR insensitivity was dependent on which serk 3 mutant allele used in the generation of double mutant serk1serk3. Our report also depicted that based on conventional brassinolide (BL) assays, the At1g27190 protein was involved in BR signaling pathway. We also showed that the triple mutant serk1-1serk3-2at1g27190 revealed a dwarf phenotype. Determining this dwarf phenotype linked to BRI1 signaling impairment needs to be further investigated.

\section{MATERIALS AND METHODS}

\section{Plant Growth Conditions}

Unless otherwise specified, plant materials used in this study were Arabidopsis thaliana ecotype Columbia as the wild type control. Freshly harvested seeds sterilization were carried out as previously described (Lindsey III et al., 2017). Once the sterilized seeds were collected, they were germinated on $0.5 x$ Murashige and Skoog medium (Duchefa) contained with 1\% sucrose. After vernalized for 2 days at $4^{\circ} \mathrm{C}$, seeds were transferred to growth chamber with the following conditions: $22^{\circ} \mathrm{C}$, light intensity of 130-150 $\mathrm{Em}^{-2} \mathrm{~s}^{-1}, 16: 8 \mathrm{~h}$, light: dark photoperiod and relative humidity of $80 \%$ as previously described conditions (Junker et al., 2015). Subsequently, 10 day-old seedlings were transplanted to soil and grown under the same environment conditions. For the selection of transgenic seedlings, 0.5x Murashige and Skoog medium (Duchefa) supplemented with 1\% sucrose and $15 \mathrm{mg} \mathrm{L}^{-1}$ phosphinothricin (PPT; Duchefa) were used.

For the hypocotyl length and root length experiment with or without BR, after vernalization for 2 days, the seeds were vertically kept in the growth chamber in the darkness for 5 days, or in the light for 7 days, respectively. Every experiment was biologically repeated three times. Images were analyzed by ImageJ software.

\section{PCR-based Genotyping}

The serk1-1, serk3-1 (Kemmerling et al., 2007; Russinova et al., 2004), bri1-301, and bri1-19 were previously described (Alonso et al., 2003; Zhang et al., 2018). PCR genotyping for single, double, and triple mutants was performed with published primers combinations (Albrecht et al., 2005, 2008).

\section{Gene Cloning and Plant Transformation}

For the production of BRI1-GFP construct, the full length BRII gene cDNA were cloned with iProof ${ }^{\mathrm{TM}}$ high-fidelity DNA polymerase and then fused to green fluorescent protein $(G F P)$ gene by primers engineered with $N c o$ I restriction sites.

To produce the BRII promoter construct, a 2-kb region upstream of the start codons of the BRI1 gene was cloned in the pGEM ${ }^{\circledR}-\mathrm{T}$ vector (Promega Corporation) with iProof ${ }^{\mathrm{TM}}$ high-fidelity DNA polymerase. The pGEM-T cloned promoters were inserted via SalI-NcoI in a modified pBluescript 
vector containing the GFP gene inserted as NcoI-BamHI fragment in front of the Tnos terminator. The entire open reading frames of BRII gene as described above were then inserted as $\mathrm{NcoI}$ fragments. The resulting full cassettes were then sub-cloned into a modified pFluar vector via SalI-SmaI (Stuitje et al., 2003). These constructs were further referred to as PBR1: BRI1-GFP.

These constructs were verified by sequencing and were electroporated in Agrobacterium tumefaciens strain C58C1 containing a disarmed C58 Ti plasmid (Koncz et al., 1989). The constructs were transformed into the serk1-3serk3-2 mutant backgrounds by the floral-dip method (Clough \& Bent, 1998). All the primers were designed and provided by Catherine Albrecht, Laboratory of Biochemistry, Wageningen University.

\section{RESULTS AND DISCUSSION}

Generation of the Double Mutant serk13serk3-2 and serk1-3serk3-2bri1-301

Based on root length assay, it was reported that the serk1-1 was weaker allele than the serk1-3, while both alleles showed a similar male sterile phenotype (Albrecht et al., 2008). Similarly, the serk3-1 allele was weaker than the serk3-2 allele in innate immunity assays but not in BRrelated assays (Chinchilla et al., 2007; Kemmerling et al., 2007). Furthermore, although the double mutant serk3-1serk4 displayed a viable dwarf stature, the double mutant serk3-2 serk4 was lethal. These data clearly indicated that the different serkl and serk 3 mutant alleles showed various level of penetrance of BR sensitivity. Additionally, we also theorized that the level of BR resistance could be increased by incorporating bril mutant allele in the double mutant serk1-3serk3-2. As a result, the double mutant serk1-3serk3-2 and triple mutant serk1-3serk3-2bri1-301 were generated. We further analyzed the BRrelated phenotypes of these two mutant lines in the root inhibition assay.

\section{BR-related Phenotypes of the Double Mutant serk1-3serk3-2 and serk1- 3serk3-2bri1-301}

Root length assay was performed to test whether the BR sensitivity of the double mutant serk1-3serk3-2 and triple mutant serk1-3serk3-2bri1-301. Seedlings were grown vertically with different BR concentrations under the light. The root lengths were subsequently photographed and analyzed. Although the serk1-3 single mutant did not show a reduced BR sensitivity (Albrecht et al., 2008), an increase in BR sensitivity even at high BR concentrations $(100 \mathrm{nM})$ could be observed in the double mutant serk1-3serk3-2, which phenocopied the BL response of the bri1-301 mutant (Figure 1) (Greene et al., 2003). It was also noteworthy the triple mutant serk13serk3-2bri1-301 did not enhance the BR insensitivity. Although we could observe a strong BR-related phenotype in the root, we did not record any increased dwarfism in the serk1serk3 double mutant as compared to the serk3-2 single mutant. Taken together, these data clearly showed that the double mutant serk1-3 serk3-2 enhanced the BR insensitivity. 


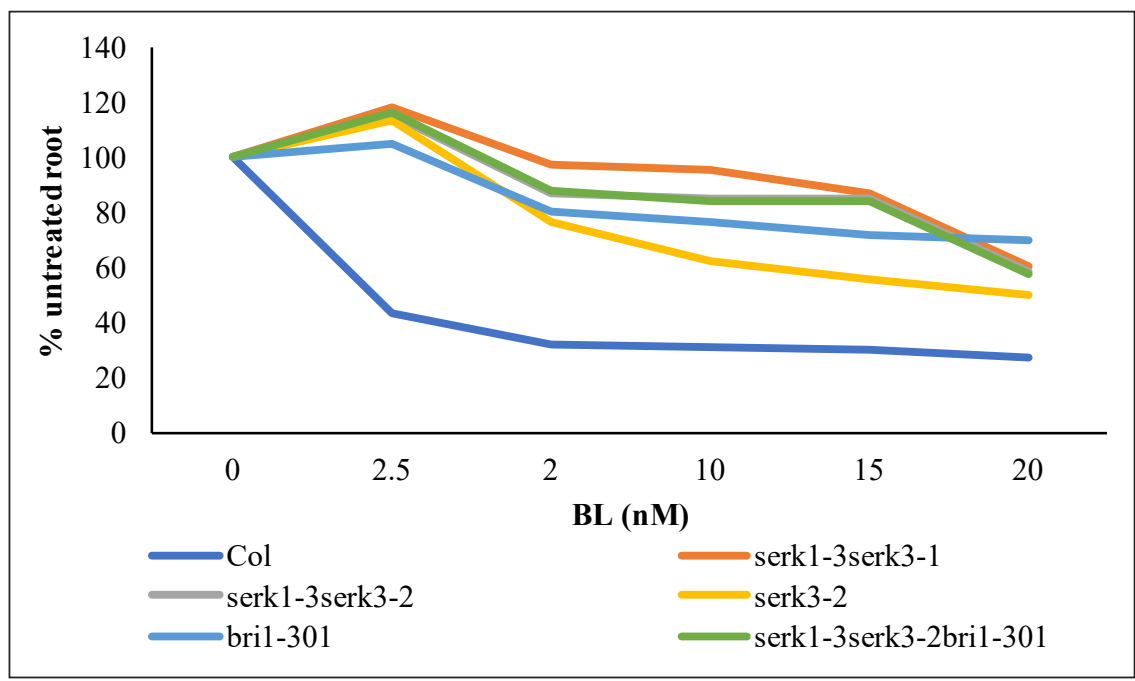

Figure 1. Root length assay of bril-301 and serk3-2 single mutants, serk1-3 in combinations with serk31, serk3-2, and serk3-2bril-301 backgrounds. Wild type Col serves as control treatment (Note. BL = Brassinolide)

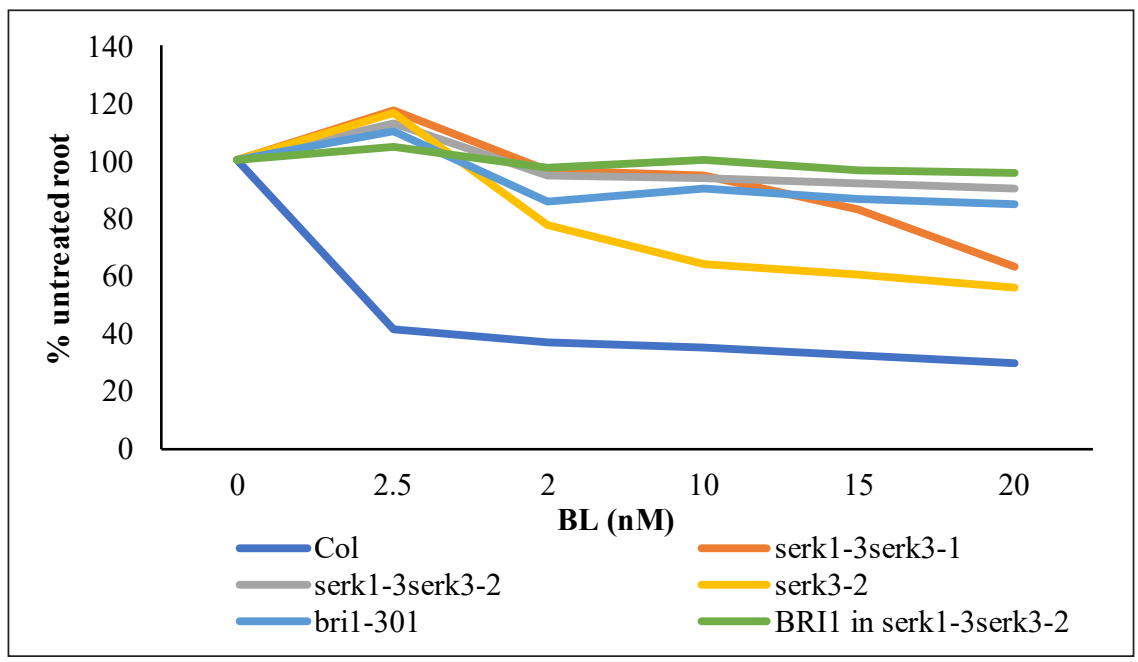

Figure 2. Root length assay of bri1-301 and serk3-2 single mutants, transgenic pBRI1::BRI1 in serk13serk3-2 background, and serk1-3 in combinations with serk3-1 and serk3-2 backgrounds. Wild type Col serves as control treatment (Note. $\mathrm{BL}=$ Brassinolide)

\section{An Alternative SERK-independent BRI1 Pathway}

It has been shown that BRI1 protein can operate in the homo-dimeric state, thus suggesting an alternative BRI1 pathway that would operate without the SERK co- receptors. This would imply that it is possible to partially or fully rescue a serk1serk 3 mutant phenotype by supplementing a transgene BRII in a serk1serk 3 mutant background. As a result, the serk1serk 3 strong double mutant was crossed with BRII 
overexpressing line. The BRII/serk1serk 3 lines were subsequently analyzed in the root inhibition assay. We used the overexpressing $B R I-G F P$ line and the bes $1-D$ mutant line, which showed an overexpression BR phenotype due the dominant positive mutation in the BES1 transcription factor, as controls. Our data presented that BRII/ serk1 serk 3 lines displayed BR insensitivity (Figure 2). This indicates that in the root the BRI1 signaling is mediated through the SERK1 and SERK3 co-receptors.

\section{Characterization of the At1g27190 Mutants}

Our protein mass spectrometry experiments demonstrated that At $\lg 27190$ participated in the same complex with BR1, SERK1, and SERK3 (data not shown). Consequently, mutant line of At $\lg 27190$ gene was obtained for characterization. The mutant Atlg27190 lines illustrated indistinguishable phenotypes compared to the wild type. Since PCR genotyping indicated that the T-DNA inserted in the Atlg27190 gene was located in kinase region, the Atlg27190 mutant gene would encode a non-functional protein lacking approximately 200 amino acids of the kinase domain (unpublished data).

We then proceeded to cross At $1 g 27190$ mutant line with the serk mutants to observe any enhancement in reported serk mutant phenotypes. Double, triple mutants were developed by crossing the At $\lg 27190$ mutants with serk1-1serk2-1serk3-1 and serk1-1serk2-1serk3-2 triple mutants. Based on the genotyping and phenotyping results, double mutants serk1-1 atlg27190, serk3-2 atlg27190, serk3-1at1g27190, triple mutants serk1-1serk3-1at1g27190 demonstrated an indistinguishable phenotype from the wild type. However, triple mutants serk1-1 1serk3-2 at lg27190 exhibited dwarf phenotype (Figure 3). Whether Atlg27190 acts in synergy with the SERK co-receptors and enhances the phenotype of the serk1serk 3 double mutant needs further confirmation.

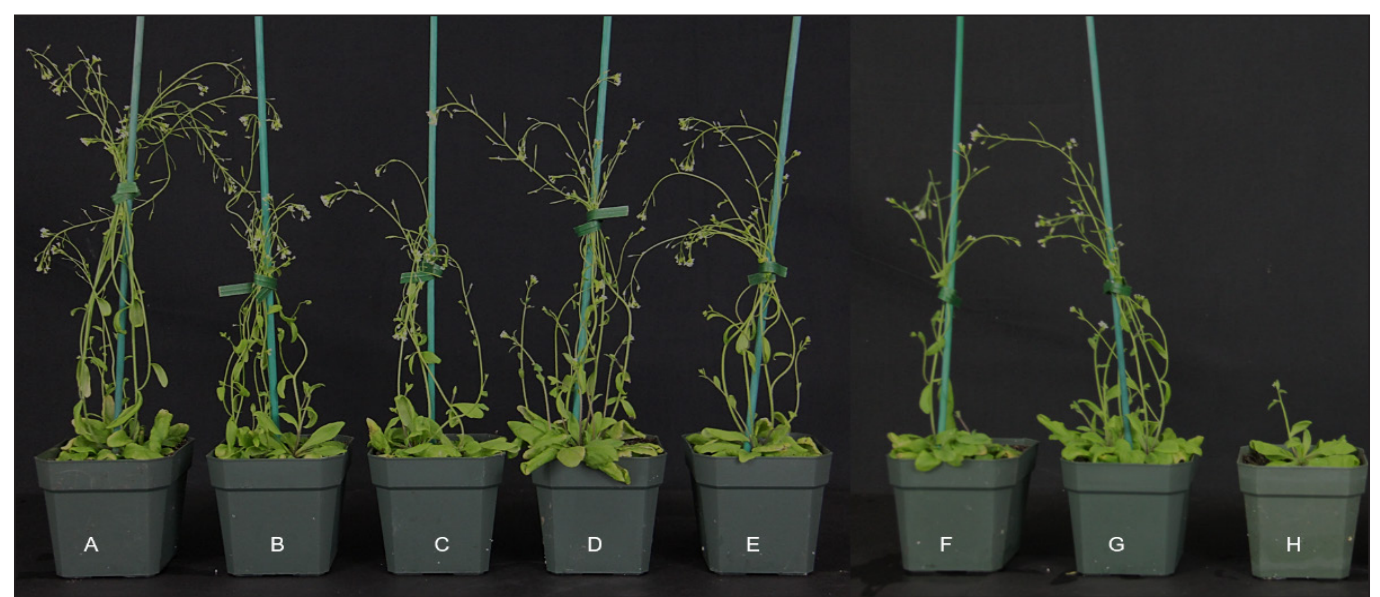

Figure 3. Phenotypic analysis of 12-week-old Arabidopsis thaliana used in this study. (A) wild type; (B) serk1-1; (C) serk3-2; (D) serk1-1 at1g27910; (E) serk3-1 at1g27910; (F) serk3-2 at1g27910; (G) serk1-1 serk3-1at1g27910; (H) serk1-1 serk3-2at1g27910, respectively. 
BR-related Phenotypes of the At1g27190 Mutants

As a first step to evaluate the involvement of At 1 g27190 in BR signaling pathway, two different alleles of single mutant At $1 g 27190$ BIL3-N616632 and BIL3-632078, were tested in the root length assay and the hypocotyl assay. In the root length assay, the Atlg27190 mutants showed the same level of BL insensitivity as the serk3-1 (Figure
4) at low (2.5 to $10 \mathrm{nM})$ concentrations of BL. In the hypocotyl length assay, it was previously reported that the serk 3 mutant alleles, serk3-1 and serk3-2, had shorter hypocotyls than the wild type when grown in dark (Li et al., 2002; Nam \& Li, 2002). In our study, the dark-grown hypocotyls of the Atlg27190 mutants were decreased compared to the control wild-type plants (Figure 5).

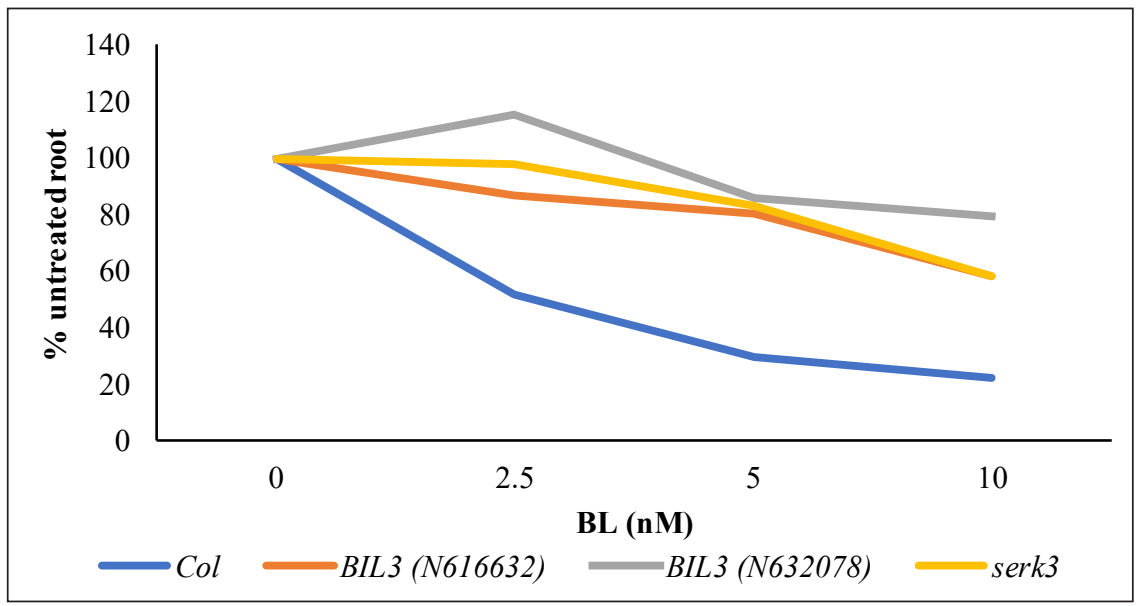

Figure 4. Root length assay of Atlg27190 T-DNA tags. Wild type Col serves as control treatment (Note. BL $=$ Brassinolide)

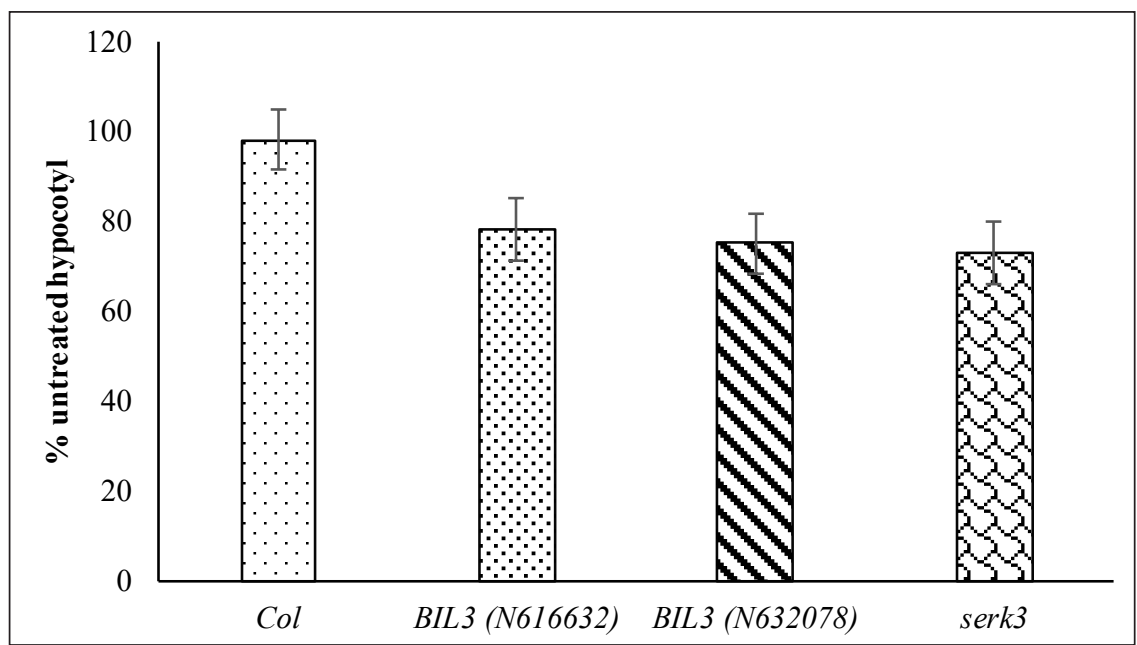

Figure 5. Hypocotyl length assay of At 1 27190 T-DNA tags. Wild type Col serves as control treatment 


\section{CONCLUSION}

Our data indicated that the double mutants serk1-3serk3-2 and serk1-3serk3-2bril-301 depicted a complete BR insensitive phenotype in the root comparable to that of a bril mutant. As a result, we propose that all the BRI1 signaling in the root is mediated through the SERK1 and SERK3 co-receptors. Our report also depicted that based on conventional BL assays, the At1g27190 protein was involved in brassinosteroid signaling and the triple mutant serk1-1serk3-2at1g27190 revealed a dwarf phenotype. Determining this dwarf phenotype linked to BRI1 signaling impairment needs to be further investigated.

\section{ACKNOWLEDGEMENT}

The author was in debt to Dr. Albrecht for her guidance.

\section{REFERENCES}

Albrecht, C., Boutrot, F., Segonzac, C., Schwessinger, B., Gimenez-Ibanez, S., Chinchilla, D., . . . Zipfel, C. (2012). Brassinosteroids inhibit pathogen-associated molecular pattern-triggered immune signaling independent of the receptor kinase BAK1. Proceedings of the National Academy of Sciences of the United States of America, 109(1), 303-308. doi: 10.1073/ pnas. 1109921108

Albrecht, C., Russinova, E., Hecht, V., Baaijens, E., \& de Vries, S. (2005). The Arabidopsis thaliana SOMATIC EMBRYOGENESIS RECEPTOR-LIKE KINASES1 and 2 control male sporogenesis. The Plant Cell, 17(12), 33373349. doi: 10.1105/tpc.105.036814

Albrecht, C., Russinova, E., Kemmerling, B., Kwaaitaal, M., \& de Vries, S. C. (2008).
Arabidopsis SOMATIC EMBRYOGENESIS RECEPTOR KINASE proteins serve brassinosteroid-dependent and -independent signaling pathways. Plant Physiology, 148(1), 611-619. doi: 10.1104/pp.108.123216

Alonso, J. M., Stepanova, A. N., Leisse, T. J., Kim, C. J., Chen, H., Shinn, P., . . . Ecker, J. R. (2003). Genome-wide insertional mutagenesis of Arabidopsis thaliana. Science, 301(5633), 653-657. doi: 10.1126/science.1086391

Belkhadir, Y., \& Chory, J. (2006). Brassinosteroid signaling: A paradigm for steroid hormone signaling from the cell surface. Science, 314(5804), 1410-1411. doi: 10.1126/ science. 1134040

Belkhadir, Y., \& Jaillais, Y. (2015). The molecular circuitry of brassinosteroid signaling. New Phytologist, 206(2), 522-540. doi: 10.1111/ nph.13269

Brandt, B., \& Hothorn, M. (2016). SERK co-receptor kinases. Current Biology, 26(6), R225-R226. doi: 10.1016/j.cub.2015.12.014

Bücherl, C. A., van Esse, G. W., Kruis, A., Luchtenberg, J., Westphal, A. H., Aker, J., ... de Vries, S. C. (2013). Visualization of BRI1 and BAK1 (SERK3) membrane receptor heterooligomers during brassinosteroid signaling. Plant Physiology, 162(4), 1911-1925. doi: $10.1104 /$ pp.113.220152

Chinchilla, D., Zipfel, C., Robatzek, S., Kemmerling, B., Nürnberger, T., Jones, J. D. G., . . . Boller, T. (2007). A flagellin-induced complex of the receptor FLS2 and BAK1 initiates plant defence. Nature, 448(7152), 497-500. doi: 10.1038/ nature 05999

Clough, S. J., \& Bent, A. F. (1998). Floral dip: A simplified method for Agrobacterium-mediated transformation of Arabidopsis thaliana. The Plant Journal, 16(6), 735-743. doi: 10.1046/j.1365313x.1998.00343.x 
Fan, M., Wang, M., \& Bai, M.-Y. (2016). Diverse roles of SERK family genes in plant growth, development and defense response. Science China Life Sciences, 59(9), 889-896. doi: 10.1007/s11427-016-0048-4

Greene, E. A., Codomo, C. A., Taylor, N. E., Henikoff, J. G., Till, B. J., Reynolds, S. H., . . Henikoff, S. (2003). Spectrum of chemically induced mutations from a large-scale reverse-genetic screen in Arabidopsis. Genetics, 164(2), 731740 .

Hutten, S. J., Hamers, D. S., Aan den Toorn, M., van Esse, W., Nolles, A., Bücherl, C. A., . . Borst, J. W. (2017). Visualization of BRI1 and SERK3/ BAK1 nanoclusters in Arabidopsis roots. PLOS One, 12(1), e0169905. doi: 10.1371/journal. pone. 0169905

Junker, A., Muraya, M. M., Weigelt-Fischer, K., AranaCeballos, F., Klukas, C., Melchinger, A. E., . . . Altmann, T. (2015). Optimizing experimental procedures for quantitative evaluation of crop plant performance in high throughput phenotyping systems. Frontiers in Plant Science, 5, 770. doi: 10.3389/fpls.2014.00770

Kang, Y. H., Breda, A., \& Hardtke, C. S. (2017). Brassinosteroid signaling directs formative cell divisions and protophloem differentiation in Arabidopsis root meristems. Development, 144(2), 272-280. doi: 10.1242/dev.145623

Karlova, R., Boeren, S., Russinova, E., Aker, J., Vervoort, J., \& de Vries, S. (2006). The Arabidopsis SOMATIC EMBRYOGENESIS RECEPTOR-LIKE KINASE1 protein complex includes BRASSINOSTEROIDINSENSITIVE1. The Plant Cell, 18(3), 626-638. doi: $10.1105 /$ tpc. 105.039412

Kemmerling, B., Schwedt, A., Rodriguez, P., Mazzotta, S., Frank, M., Qamar, S. A., . . Nürnberger, T. (2007). The BRI1-associated kinase 1, BAK1, has a brassinolide-independent role in plant cell- death control. Current Biology, 17(13), 11161122. doi: 10.1016/j.cub.2007.05.046

Kinoshita, T., Caño-Delgado, A., Seto, H., Hiranuma, S., Fujioka, S., Yoshida, S., \& Chory, J. (2005). Binding of brassinosteroids to the extracellular domain of plant receptor kinase BRI1. Nature, 433(7022), 167-171. doi: 10.1038/nature03227

Koncz, C., Martini, N., Mayerhofer, R., KonczKalman, Z., Körber, H., Redei, G. P., \& Schell, J. (1989). High-frequency T-DNA-mediated gene tagging in plants. Proceedings of the National Academy of Sciences of the United States of America, 86(21), 8467-8471. doi: 10.1073/ pnas.86.21.8467

Li, J., Wen, J., Lease, K. A., Doke, J. T., Tax, F. E., \& Walker, J. C. (2002). BAK1, an Arabidopsis LRR receptor-like protein kinase, interacts with BRI1 and modulates brassinosteroid signaling. Cell, 110(2), 213-222. doi: 10.1016/S00928674(02)00812-7

Lindsey III, B. E., Rivero, L., Calhoun, C. S., Grotewold, E., \& Brkljacic, J. (2017). Standardized method for high-throughput sterilization of Arabidopsis seeds. Retrieved May 10, 2020, from https://www.ncbi.nlm.nih.gov/ pmc/articles/PMC5752416/

Liu, J., Li, J., \& Shan, L. (2020). SERKs. Current Biology, 30(7), R293-R294. doi: 10.1016/j. cub.2020.01.043

Nam, K. H., \& Li, J. (2002). BRI1/BAK1, a receptor kinase pair mediating brassinosteroid signaling. Cell, 110(2), 203-212. doi: 10.1016/S00928674(02)00814-0

Russinova, E., Borst, J.-W., Kwaaitaal, M., CañoDelgado, A., Yin, Y., Chory, J., \& de Vries, S. C. (2004). Heterodimerization and endocytosis of Arabidopsis brassinosteroid receptors BRI1 and AtSERK3 (BAK1). The Plant Cell, 16(12), 3216-3229. doi: 10.1105/tpc.104.025387 
Santiago, J., Henzler, C., \& Hothorn, M. (2013). Molecular mechanism for plant steroid receptor activation by somatic embryogenesis co-receptor kinases. Science, 341(6148), 889-892. doi: 10.1126/science. 1242468

Stuitje, A. R., Verbree, E. C., Van Der Linden, K. H., Mietkiewska, E. M., Nap, J. P., \& Kneppers, T. J. (2003). Seed-expressed fluorescent proteins as versatile tools for easy (co)transformation and high-throughput functional genomics in Arabidopsis. Plant Biotechnology Journal, 1(4), 301-309.

Sun, Y., Han, Z., Tang, J., Hu, Z., Chai, C., Zhou, B., \& Chai, J. (2013). Structure reveals that BAK1 as a co-receptor recognizes the BRI1-bound brassinolide. Cell Research, 23(11), 1326-1329. doi: 10.1038/cr.2013.131

Sun, C., Yan, K., Han, J. T., Tao, L., Lv, M. H., Shi, T., . . . Li, J. (2017). Scanning for new BRI1 mutations via TILLING analysis. Plant Physiology, 174(3), 1881-1896. doi: 10.1104/ pp.17.00118

van Esse, G. W., Ten Hove, C. A., Guzzonato, F., van Esse, H. P., Boekschoten, M., Ridder, L., . . . de Vries, S. C. (2016). Transcriptional analysis of serk1 and serk3 coreceptor mutants. Plant Physiology, 172(4), 2516-2529. doi: 10.1104/ pp. 16.01478

van Esse, W., van Mourik, S., Albrecht, C., van Leeuwen, J., \& de Vries, S. (2013). A mathematical model for the coreceptors
SOMATIC EMBRYOGENESIS RECEPTORLIKE KINASE1 and SOMATIC EMBRYOGENESIS RECEPTOR-LIKE KINASE3 in BRASSINOSTEROID INSENSITIVE1-mediated signaling. Plant Physiology, 163(3), 1472-1481. doi: 10.1104/ pp.113.222034

van Esse, G. W., van Mourik, S., Stigter, H., ten Hove, C. A., Molenaar, J., \& de Vries, S. C. (2012). A mathematical model for BRASSINOSTEROID INSENSITIVE1-mediated signaling in root growth and hypocotyl elongation. Plant Physiology, 160(1), 523-532. doi: 10.1104/ pp.112.200105

Wang, X., Goshe, M. B., Soderblom, E. J., Phinney, B. S., Kuchar, J. A., Li, J., . . Clouse, S. D. (2005). Identification and functional analysis of in vivo phosphorylation sites of the Arabidopsis BRASSINOSTEROID-INSENSITIVE1 receptor kinase. The Plant Cell, 17(6), 1685-1703. doi: 10.1105/tpc.105.031393

Zhang, X., Zhou, L., Qin, Y., Chen, Y., Liu, X., Wang, M., . . . Li, J. (2018). A temperature-sensitive misfolded bri1-301 receptor requires its kinase activity to promote growth. Plant Physiology, 178(4), 1704-1719. doi: 10.1104/pp.18.00452

Zheng, L., Ma, J., Mao, J., Fan, S., Zhang, D., Zhao, C., . . . Han, M. (2018). Genome-wide identification of SERK genes in apple and analyses of their role in stress responses and growth. BMC Genomics, 19(1), 962-962. doi: 10.1186/s12864-018-5342-1 